\title{
O-5S quantitative real-time PCR: a new diagnostic tool for laboratory confirmation of human onchocerciasis
}

Solomon A. Mekonnen ${ }^{1,2^{*}}$, Marcus Beissner ${ }^{3 \dagger}$, Malkin Saar ${ }^{3}$, Solomon Ali ${ }^{1}$, Ahmed Zeynudin ${ }^{1}$, Kassahun Tesfaye ${ }^{2}$, Mulatu G. Adbaru' ${ }^{1}$ Florian Battke ${ }^{4}$, Sven Poppert ${ }^{5}$, Michael Hoelscher ${ }^{3,6}$, Thomas Löscher ${ }^{3}$, Gisela Bretzel ${ }^{3}$ and Karl-Heinz Herbinger ${ }^{3}$

\begin{abstract}
Background: Onchocerciasis is a parasitic disease caused by the filarial nematode Onchocerca volvulus. In endemic areas, the diagnosis is commonly confirmed by microscopic examination of skin snip samples, though this technique is considered to have low sensitivity. The available melting-curve based quantitative real-time PCR (qPCR) using degenerated primers targeting the O-150 repeat of $O$. volvulus was considered insufficient for confirming the individual diagnosis, especially in elimination studies. This study aimed to improve detection of O. volvulus DNA in clinical samples through the development of a highly sensitive QPCR assay.

Methods: A novel hydrolysis probe based qPCR assay was designed targeting the specific sequence of the $O$. volvulus O-5S rRNA gene. A total of 200 clinically suspected onchocerciasis cases were included from Goma district in South-west Ethiopia, from October 2012 through May 2013. Skin snip samples were collected and subjected to microscopy, O-150 qPCR, and the novel O-5S qPCR.

Results: Among the 200 individuals, 133 patients tested positive (positivity rate of 66.5\%) and 67 negative by O-5S qPCR, 74 tested positive by microscopy (37.0\%) and 78 tested positive by O-150 qPCR (39.0\%). Among the 133 O-5S qPCR positive individuals, microscopy and O-150 qPCR detected 55.6 and 59.4\% patients, respectively, implying a higher sensitivity of O-5S qPCR than microscopy and O-150 qPCR. None of the 67 individuals who tested negative by O-5S qPCR tested positive by microscopy or O-150 qPCR, implying 100\% specificity of the newly designed O-5S qPCR assay.

Conclusions: The novel O-5S qPCR assay is more sensitive than both microscopic examination and the existing O-150 qPCR for the detection of $O$. volvulus from skin snip samples. The newly designed assay is an important step towards appropriate individual diagnosis and control of onchocerciasis.
\end{abstract}

Keywords: Confirmation, Microscopy, Molecular diagnosis, Onchocerciasis, O-5S qPCR, O-150 qPCR, Ethiopia

\section{Background}

Onchocerciasis, also referred to as "river blindness," is caused by the filarial nematode Onchocerca volvulus, which is transmitted to humans by black flies (Simulium spp.) in endemic countries of Africa, including Ethiopia, three countries in Latin America, and in Yemen. This parasitic

\footnotetext{
* Correspondence: selemonye@gmail.com

'Equal contributors

'Department of Medical Laboratory Sciences and Pathology, College of Health Sciences, Jimma University, Jimma, Ethiopia

${ }^{2}$ Department Microbial, Cellular and Molecular Biology, Addis Ababa

University, Addis Ababa, Ethiopia

Full list of author information is available at the end of the article
}

disease was listed by the World Health Organization (WHO) as one of the eighteen neglected tropical diseases [1-4]. Onchocerciasis constitutes a major public health problem in affected regions, as an estimated 37 million persons are infected with $O$. volvulus worldwide, with the vast majority of them living in East and West Africa. As this disease is endemic in some of the world's poorest areas, it had a major impact on the economic and social aspects of affected communities [5-7]. Ethiopia is one of the countries with a high burden of onchocerciasis, where the disease has spread widely to regions that were previously non-endemic [8]. In 1998, the "African Program for Onchocerciasis 
Control" sponsored the first nationwide epidemiological mapping of onchocerciasis in Ethiopia. Following the results of that study, most areas of Central, South, and West Ethiopia were defined endemic [9].

To avoid severe progression of the disease, early detection and treatment are essential. A clinically suspected $O$. volvulus infection can be confirmed by different laboratory methods: microscopic detection of microfilariae (mf) from skin snip samples (SSS) following incubation in normal saline; detection of the major sperm protein 2 (MSP2) by dipstick test; detection of recombinant antigens by means of a luciferase immune precipitation system (QLIPS); and rapid DNA detection test strips; and polymerase chain reaction (PCR) [10-12]. The microscopic examination is cheap and easy to perform. However, this method has several limitations, as its sensitivity depends on the location where skin snips were taken, $\mathrm{mf}$ density, stage of disease, previous treatment, and especially on the qualifications of staff performing tests [13-16].

Various PCR-based assays were described for the detection of different larval stages of O. volvulus [17-22]. For more than a decade, a conventional gel-based PCR applying degenerated primers amplifying the O. volvulus-specific $\mathrm{O}$ 150 tandem repeat [23-25] was often used to confirm onchocerciasis from SSS [14, 26, 27]. In 2011, Fink et al. described a melting curve based quantitative real-time PCR (qPCR) which also used degenerated primers to detect a 154 bp amplicon of the O-150 repeat (O-150 qPCR) [28]. However, the long PCR amplicon may lead to limited sensitivity, and the lack of a hybridization probe may reduce the specificity of this assay [29]. In recent years, Lloyd et al. [14] and Golden et al. [30] showed the importance of PCR for the detection of $O$. volvulus.

In Ethiopia, cases of onchocerciasis are routinely diagnosed by microscopic examination of SSS, which has limited sensitivity especially in samples with low $\mathrm{mf}$ density and is therefore not sufficiently reliable for programmatic monitoring and evaluation of the effects of the WHO recommended mass drug administration [31]. The objective of this study was to develop and validate a novel hydrolysis probe-based qPCR targeting the $5 \mathrm{~S}$ rRNA gene of $O$. volvulus (O-5S qPCR) with enhanced sensitivity for the detection of $O$. volvulus DNA from SSS of clinically diagnosed patients from Ethiopia. The diagnostic validity of the novel qPCR assay was compared with direct microscopy and the established O-150 qPCR. The study furthermore compared socio-demographic data, potential risk factors for onchocerciasis, clinical symptoms and medical histories with the laboratory results.

\section{Methods}

\section{Study area and inclusion criteria}

The study was conducted in Goma district, which has a population of approximately 213,000 inhabitants. Goma is one of the 13 districts in Jimma zone, situated in Oromia region in Ethiopia [32]. Goma district has 36 rural and three urban "kebeles" (i.e. smallest administrative unit in Ethiopia). The number of study participants was calculated in proportion to the total number of inhabitants in each of these kebeles. The following numbers of study participants were included from four randomly chosen kebeles: Dedessa $(n=28)$, Omo Gobu $(n=52)$, Belfo Konche $(n=84)$ and Kilole Qirqir $(n=36)$. Individuals living in the study area with any form of the following clinical manifestation(s) were included in the present study: papular rash, scarring "leopard skin" (i.e. spotted depigmentation of the skin) and skin itching around the buttocks. Patients who received ivermectin treatment during the past six months were excluded.

\section{Study design and data collection}

In this cross-sectional study, data from 200 clinically suspected onchocerciasis individuals were collected from October 2012 through May 2013. After written informed consent was obtained, study participants were interviewed by trained study nurses to record socio-demographic data, potential risk factors for onchocerciasis and medical history by pre-designed and pre-tested questionnaires. Data were completely pseudonymized and transferred to an Excel-based database (Microsoft, Redmond, WA, USA). SSS were collected from either side of the buttocks by senior medical laboratory technologists according to routine standardized procedures [1].

\section{Microscopy}

The collected SSS was transferred to one cavity of a 96well plate, leaving blank cavities between samples to avoid cross contamination. Three drops of phosphate buffered saline (PBS, Sigma-Aldrich, Bangalore, India) were added to allow complete release of $\mathrm{mf}$ during a four $\mathrm{h}$ incubation period. The samples were sealed by Titer Tops sealing film for microplates (Sigma-Aldrich) and transported to a laboratory of health centers nearby the study areas for wet mount microscopic examination. There, PBS from SSS was transferred to microscopic slides for the detection of O. volvulus $\mathrm{mf}$ with light microscopy under 10 - and 40fold objectives by experienced senior laboratory technologists. The remaining samples were stored in the respective 96-well plates, transported within $24 \mathrm{~h}$ to the Department of Medical Laboratory Sciences and Pathology of Jimma University, and there immediately deep-frozen at $-20{ }^{\circ} \mathrm{C}$ until shipment by courier service to the Department for Infectious Diseases and Tropical Medicine (DITM) of the Ludwig-Maximilians University in Munich, Germany.

\section{0-5S qPCR assay}

Target sequence and primer design

A qPCR assay was developed for the detection of $O$. volvulus from human SSS. After an extensive database search 
(GenBank [PubMed, NCBI]) and sequence analyses (DNASIS Max software [MiraiBio, San Francisco, CA]), an intergenic spacer region of the O. volvulus 5S rRNA gene was chosen as a target for PCR amplification. Primers and a hydrolysis probe (MWG Eurofins, Ebersberg, Germany) for amplification of this $O$. volvulus-specific intergenic spacer region of the $5 \mathrm{~S}$ rRNA gene were designed by alignment of $5 \mathrm{~S}$ rRNA gene sequences (DNASIS Max) retrieved from GenBank [33] from closely related filarial nematodes potentially contaminating the SSS from human skin by primer 3 . Specificity of the primers for O. volvulus was confirmed in silico using the basic local alignment search tool (BLAST, GenBank, NCBI). The selected 5S rRNA target, primers and hydrolysis probe, and alignments of the target region from O. volvulus with Wuchereria bancrofti, Brugyia malayi, Loa loa, Mansonella streptocerca and Plasmodium falciparum are provided in Additional file 1.

\section{O-5S qPCR standard}

A PCR standard template was generated by gene synthesis of the target sequence and subsequent cloning to the $\mathrm{pEX}$ A2 vector (MWG, Ebersberg, Germany). The vector was transformed into E. coli (JM109, Zymo, Freiburg, Germany) and the transformation mix was plated on LB agar plates with $100 \mathrm{mg} / \mathrm{l}$ ampicillin (Carl Roth, Karlsruhe, Germany) and cultivated at $37{ }^{\circ} \mathrm{C}$ overnight. By selection of one suitable colony, a master cryo culture was obtained and stored in RotiStore tubes (Carl Roth). From this master cryo culture, a $3 \mathrm{ml}$ LB liquid culture with $100 \mathrm{mg} / \mathrm{l}$ ampicillin (Carl Roth) was grown over night. The plasmid DNA was extracted with a commercial plasmid extraction kit, according to the manufacturer's instruction (HiYield Plasmid Mini kit; Süd-Laborbedarf, Gauting, Germany). The cloned plasmid sequence was confirmed by direct DNA sequencing as previously described [34]. The purity of extracted plasmid DNA was assessed by photometry on a BioPhotometer plus (Eppendorf, Wesseling-Berzdorf, Germany) and agarose gel-electrophoresis on a $1 \%$ TAE gel. The plasmid solution was quantified by a dsDNA fluorescence quantification kit (Qubit, Life Technologies, Karlsruhe, Germany), according to the manufacturer's instruction. The number of fragments per $\mu \mathrm{l}$ was calculated and aliquots of the plasmid solution, diluted to the desired fragment numbers, were used for determination of the lower limit of detection (LOD, i.e. lowest template concentration rendering positive amplification of $95 \%$ of samples) [29].

\section{0-5S qPCR protocol}

The primers for O-5S qPCR were as described in Table 1. The reaction mixture (total volume: $20 \mu \mathrm{l}$ ) contained $8.6 \mu \mathrm{l}$ molecular grade $\mathrm{H}_{2} \mathrm{O}$ (Carl Roth), $4 \mu \mathrm{l}$ 5-fold PCR buffer qPCR Mix Plus (Solis BioDyne, Tartu, Estonia), $2 \mu$ l 10-fold Exo IPC Mix (TaqMan exogenous internal positive control reagent; ThermoFisher Scientific, Darmstadt, Germany),
Table 1 Sequences and nucleotide positions of primers and the hydrolysis probe, including the corresponding amplicon sizes of the O-5S qPCR

\begin{tabular}{llllc}
\hline Test & Primer/probe $^{\mathrm{a}}$ & Sequence $\left(5^{\prime}-3^{\prime}\right)^{\mathrm{b}}$ & $\begin{array}{l}\text { Nucleotide } \\
\text { position }^{c}\end{array}$ & $\begin{array}{c}\text { Amplicon } \\
\text { size }(\mathrm{bp})\end{array}$ \\
\hline O-5S & Ov5S-F & GAGGTAATTGAATGT & $74-96$ & 84 \\
qPCR & & TTCTGCCC & & \\
& Ov5S-R & TGTTGTCCCGCTCATGC & $158-142$ & \\
& Ov5S-P & FAM-AGTTTCGACTGCT & $99-124$ & \\
& & GTGGCTTGAAGCG-BHQ1 & & \\
\hline
\end{tabular}

${ }^{\mathrm{a}} \mathrm{F}$, forward primer; $\mathrm{R}$, reverse primer; $\mathrm{P}$, hydrolysis probe (TibMolBiol, Berlin, Germany)

${ }^{b}$ Hydrolysis probe with Fluorescein (FAM) and BlackHole Dark Quencher (BHQ) ${ }^{c}$ Nucleotide positions are provided for the intergenic spacer region of the $5 \mathrm{~S}$ ribosomal RNA gene of Onchocerca volvulus (GenBank: U31643.1)

$1 \mu \mathrm{l}$ of each primer and probe $(10 \mu \mathrm{M}), 0.4 \mu \mathrm{l} 50$-fold Exo IPC DNA (ThermoFisher Scientific) and $2 \mu \mathrm{l}$ DNA template. The amplification was performed at $95{ }^{\circ} \mathrm{C}$ for $15 \mathrm{~min}$, followed by 45 cycles of $95{ }^{\circ} \mathrm{C}$ for $15 \mathrm{~s}, 56{ }^{\circ} \mathrm{C}$ for $20 \mathrm{~s}$ and detection at $72{ }^{\circ} \mathrm{C}$ for $20 \mathrm{~s}$ on a Bio-Rad CFX-96 real-time PCR machine (Bio-Rad, Munich, Germany) and included negative extraction, negative "no template", positive run controls as well as the internal positive controls (IPC).

\section{Specificity and sensitivity of 0-5S qPCR}

Specificity of the assay was assessed by using 20 O-150 PCR positive ("must detect") and $20 \mathrm{O}-150$ PCR negative ("must not detect") O. volvulus clinical DNA extracts from SSS collected in a previous study [35], confirmed DNA extracts from Dirofilaria immitis $(n=3)$, D. repens $(n=1)$, Loa loa $(n=1)$ and Mansonella streptocerca $(n=1)$. The analytical sensitivity of the O-5S qPCR assay was determined as LOD by using 10-fold serial dilutions of O-5S plasmid standards with known copy numbers. Intra- and inter-assay variability were assessed by testing each sample in quadruplicate within one 96-well plate, repeated on three different days. Variability was judged low if the maximum cycle threshold variation range $\left(\mathrm{C}_{\mathrm{q}}\right.$-range $\mathrm{max}_{\max }$; i.e. range of $\mathrm{C}_{\mathrm{q}}$-values of samples tested in the same dilution) was $\leq 0.5$ (intra-assay) and $\leq 1.0$ (inter-assay).

\section{0-150 melting curve based qPCR}

The melting curve based O-150 qPCR assay was conducted according to the protocol described by Fink et al. [28] on a Bio-Rad CFX-96 real-time PCR machine (Bio-Rad) and included negative extraction, negative "no template" and positive run controls.

\section{0-150 hybridization probe based qPCR assay}

The O-150 hybridization probe based qPCR assay was performed as described by Golden et al. 2016 [30] with minor modifications as the original $\mathrm{qPCR}$ protocol did not lead to any amplicons. Briefly, the sequences of primers and probe were: OvFWD 5'-TGT GGA AAT TCA CCT AAA TAT G- 
3', OvREV 5'-AAT AAC TGA TGA CCT ATG ACC-3' (Eurofins Genomics, Ebersberg, Germany), and OvProbe 5' 6FAM-TAG GAC CCA ATT CGA ATG TAT GTA CCCMGBNFQ-3' (minor groove binding TaqMan ${ }^{\circ}$ Probe \#5208995 P/N 4316033, Applied Biosystems, Inchinnan, UK). The qPCR assay was designed to amplify the O-150 repeat of O. volvulus using $7.5 \mu$ SuperHot Master Mix $(2 \times)$ (BIORON, Ludwigshafen, Germany), $0.1 \mu \mathrm{l}$ of $100 \mu \mathrm{M}$ forward primer, $0.1 \mu \mathrm{l}$ of $100 \mu \mathrm{M}$ reverse primer, $0.1 \mu \mathrm{l}$ of $100 \mu \mathrm{M}$ probe, and 7.2 $\mu \mathrm{l}$ DNA extract/negative control (nuclease free water), positive control (O150 plasmid standard). Finally, the O-150 qPCR was run in a total volume of $15 \mu \mathrm{l}$ with an initial denaturation at $94{ }^{\circ} \mathrm{C}$ for $2 \mathrm{~min}$, [denaturation at $94{ }^{\circ} \mathrm{C}$ for $15 \mathrm{~s}$, hybridization at $49{ }^{\circ} \mathrm{C}$ for $30 \mathrm{~s}$, and elongation at $60{ }^{\circ} \mathrm{C}$ for $\left.2 \mathrm{~min}\right] \times 45$ cycles. All test or control samples were run in a single well and analyzed using an Illumina Eco Real-time PCR system. An O-150 plasmid standard (Genbank accession number J04659.1) was generated by gene synthesis (Eurofins Genomics). The maximum quantification cycle for a positive result was set to 37 according to the lowest detectable plasmid standard concentration. This assay was performed in an observer-blinded way without knowledge of microscopy or other PCR results.

\section{Comparison of 0-150 and 0-5S qPCR assays Sample transport}

The SSS were thawed and transferred into $2 \mathrm{ml}$ screw cap tubes (Sarstedt, Nümbrecht, Germany) containing $700 \mu \mathrm{l}$ cell lysis buffer (CLS, Qiagen, Hilden, Germany) to allow transport at ambient temperature without DNA degradation by courier service to DITM.

\section{DNA extraction}

The dsDNA of O. volvulus was extracted using the Puregene DNA extraction tissue kit (Qiagen) using enzymatic lysis as described elsewhere [35] at DITM. Briefly, SSS were inactivated at $95{ }^{\circ} \mathrm{C}$ for $15 \mathrm{~min}$. Subsequently, samples were incubated overnight at $55{ }^{\circ} \mathrm{C}$ in $700 \mu \mathrm{l} \mathrm{CLS}$ enriched with proteinase $\mathrm{K}$ (Sigma-Aldrich) to a final concentration of $300 \mu \mathrm{g} / \mathrm{ml}$. The proteinase $\mathrm{K}$ was inactivated at $95{ }^{\circ} \mathrm{C}$ for $15 \mathrm{~min}$. After the specimens were cooled to ambient temperature, lysozyme (Qiagen) was added to a final concentration of $250 \mu \mathrm{g} / \mathrm{ml}$ and specimens were incubated at $37{ }^{\circ} \mathrm{C}$ for one h. Finally, the DNA pellets were resuspended in $200 \mu$ DNA hydration solution (Qiagen). DNA extracts were stored at $4{ }^{\circ} \mathrm{C}$ (short-term, up to one week) or $-20{ }^{\circ} \mathrm{C}$ (long-term storage) until further procession by PCR.

\section{qPCR assays}

At DITM DNA extracts from SSS were subjected to O-150 qPCR and to the novel O-5S qPCR assays by independent and blinded laboratory technicians who were unaware of the microscopic results.

\section{Statistical analyses}

All data were stored in a Microsoft Excel database (Microsoft). Bivariate tests (chi-square tests including Fisher's exact test and McNemar chi-square test for matched pairs of samples with categorical test results), multivariate logistic regression, $\mathrm{t}$-tests as parametric test, and estimation of standard error of proportion (to calculate 95\% confidence intervals, 95\% CI, of categorical test results) were conducted using Epilnfo, version 3.3.2. (Centers for Disease Control and Prevention, Atlanta, GA) and Stata software, version 9.0. (Stata Corporation, College Station, TX). Significant differences were defined as $p$ values below 0.05 or as non-overlapping 95\% CI of proportions. The sensitivity and specificity of microscopy and O-150 qPCR were calculated by comparing these results with those of $\mathrm{O}-5 \mathrm{~S}$ qPCR as a reference test.

\section{Results}

\section{Novel 0-5S qPCR results}

Out of 266 individuals with clinically suspected onchocerciasis, 200 (75.19\%) fulfilled the inclusion criteria and composed the study population. Among these, 133 (66.50\%) tested positive by the newly developed O-5S qPCR assay. Interestingly, none of the individuals who tested negative by the novel O-5S qPCR tested positive by microscopy or by O-150 qPCR assay (Table 2).

\section{Microscopy results}

Among the 200 individuals comprising the study population, microscopic examination of SSS detected O. volvulus $\mathrm{mf}$ in 74 individuals, corresponding to a positivity rate of $37.00 \%$. These individuals tested positive by the newly developed assay, O-5S qPCR, which corresponds to a sensitivity of $55.64 \%$ (95\% CI: 47.20-64.08\%), and none of the individuals who tested positive by microscopy tested negative by $\mathrm{O}-5 \mathrm{~S}$ qPCR, which corresponds to a specificity of $100 \%$. The probability for positive microscopy results to be true positive (positive predictive value) was $100 \%$, and for negative results to be true negative (negative predictive value) was $53.17 \%$ (95\% CI: 44.46-61.89) (Table 2).

\section{0-150 qPCR results}

Among the 200 individuals clinically suspected for onchocerciasis, the O-150 qPCR assay by Fink et al. detected DNA of $O$. volvulus in 78 patients, which corresponds to a positivity rate of $39.00 \%$. All these patients tested positive by O-5S qPCR, which corresponds to a sensitivity of $59.40 \%$ (95\% CI: 50.28-67.02\%), with none of these individuals testing negative by $\mathrm{O}-5 \mathrm{~S}$ qPCR, which corresponds to a specificity of $100 \%$. The probability for positive O-150 qPCR results to be true positive (positive predictive value) was $100 \%$, and for negative results to be true negative (negative predictive value) was 54.92\% (95\% CI: $46.09-$ 63.75) (Table 2). 
Table 2 Comparison of microscopy, O-150 qPCR assay and O-5S qPCR assay for the diagnosis of onchocerciasis

\begin{tabular}{|c|c|c|c|c|c|}
\hline \multicolumn{2}{|l|}{ Variable } & \multirow[t]{2}{*}{ Study population $n(\%)$} & \multicolumn{2}{|l|}{ O-5S qPCR } & \multirow[t]{2}{*}{$P$-value } \\
\hline & & & Positive casesn (\%) & Negative controlsn (\%) & \\
\hline Sample size & Patients & $200(100)$ & $133(100)$ & $67(100)$ & na \\
\hline \multirow[t]{2}{*}{ Microscopy } & Positive & $74(37.00)$ & $74(55.64)$ & $0(0)$ & \multirow[t]{2}{*}{$<0.01^{\mathrm{a} *}$} \\
\hline & Negative & $126(63.00)$ & $59(44.36)$ & $67(100)$ & \\
\hline \multirow[t]{2}{*}{ O-150 qPCR } & Positive & $79(39.50)$ & $79(59.40)$ & $0(0)$ & \multirow[t]{2}{*}{$<0.01^{\mathrm{a} x}$} \\
\hline & Negative & $121(60.50)$ & $54(40.60)$ & $67(100)$ & \\
\hline \multirow[t]{4}{*}{ Microscopy + O-150 qPCR } & Negative + negative & $109(54.50)$ & $42(31.58)$ & $67(100)$ & na \\
\hline & Negative + positive & $17(8.50)$ & $17(12.78)$ & $0(0)$ & na \\
\hline & Positive + negative & $12(6.00)$ & $12(9.02)$ & $0(0)$ & na \\
\hline & Positive + positive & $62(31.00)$ & $62(46.62)$ & $0(0)$ & na \\
\hline
\end{tabular}

${ }^{a}$ McNemar's chi-square test for matched pairs: $X^{2}=59.00$ (microscopy and O-5S qPCR); $X^{2}=54.00$ (O-150 qPCR and O-5S qPCR)

*Significant differences were defined as $P$-values $<0.05$

Abbreviation: na not applicable

From the total of 200 SSS collected among individuals clinically suspected for onchocerciasis, the O-150 qPCR assay by Golden et al. detected DNA of O. volvulus in 33 of them, which corresponds to a positivity rate of $16.5 \%$. Of these 33 individuals, only 23 individuals tested positive by microscopy, the O-150 qPCR assay by Fink et al. assay and our newly developed O-5S qPCR assay, respectively. Unexpectedly, ten individuals that tested positive in the assay by Golden et al. tested negative with all other three diagnostic methods (Additional file 2: Table S1). Therefore, these results could not be used as reference standard.

Performance characteristics of the novel 0-5S qPCR assay In silico analysis of the novel primers/probe revealed $100 \%$ specificity for O. volvulus among human pathogenic filariae, which excluded amplification of human DNA. Testing of "must detect $\mathrm{O}-5 \mathrm{~S}$ samples" revealed positive amplification results for 20/20 (100\%) O. volvulus DNA extracts, while all other "must not detect O-5S samples" remained negative. Therefore, the specificity of the novel O-5S qPCR was $100 \%$. The LOD was six copies of the target sequence of $O$. volvulus. Intra- and inter-assay variabilities were low. The calibration curve is provided in Additional file 3: Figure S1. Furthermore, the novel O-5S qPCR assay does not amplify the DNA of Mansonella streptocerca, a closely related filarial parasite (Fig. 1).

\section{Concordance rate}

Among the 133 individuals tested positive by O-5S qPCR, $61(45.86 \%)$ had a positive microscopy result. Out of the 78 individuals tested positive by $\mathrm{O}-150 \mathrm{qPCR}, 13$ individuals $(9.77 \%)$ had a positive microscopy result, 17 individuals (12.78\%) had a positive result only with O-150 qPCR, and $42(31.58 \%)$ had a negative result with both tests. The concordance rate between microscopy and O-150 qPCR was $77.44 \%$ (103/133) among individuals tested positive by $\mathrm{O}-5 \mathrm{~S}$ qPCR. Among all 67 individuals tested negative by $\mathrm{O}-5 \mathrm{~S}$ qPCRs, both tests resulted in negative results, corresponding to a concordance rate between microscopy and O- 150 qPCR of $100 \%$ (67/67). In the study population of 200 patients, the overall concordance rate between microscopy and O-150 qPCR was 85\% (170/200). Between microscopy and $\mathrm{O}-5 \mathrm{~S}$ qPCR, the concordance rate was $70.5 \%(141 / 200)$, and between O-150 qPCR and O-5S qPCR, 72.5\% (145/200) (Table 2). Of note, there is no correlation between both assays (Additional file 4: Figure S2).

\section{Diagnostic yield}

The introduction of the O-150 qPCR to the already widely performed microscopy for the diagnosis of onchocerciasis has been shown in this study to provide an additional diagnostic yield of $13 \%$ (17/133 cases). By replacing microscopy with O-150 qPCR, the additional diagnostic yield would have been $3 \%$ (4/133 cases) in this study. Among the 133 individuals tested positive by O-5S qPCR, 91 (68.42\%) tested positive by microscopy or O-150 qPCR. By performing the novel O-5S qPCR, an additional diagnostic yield of $42(31.58 \%)$ was achieved (Table 2).

\section{Socio-demographics}

Among the individuals who tested positive by the newly designed O-5S qPCR assay, the proportion of females (22.56\%) was not significantly $\left(\chi^{2}=0.07, d f=1, P=0.79\right)$ different from those tested negative $(20.90 \%)$. The median age was 32 years among individuals who tested positive, whereas it was 15 years among those tested negative. The proportion of age group 30-72 years among those who tested positive (56.39\%) was significantly $\left(\chi^{2}=9.85, d f=1, P<0.01\right)$ higher than among those who tested negative (32.84\%). The proportion of farmers among individuals tested positive (70.68\%) was significantly $\left(\chi^{2}=22.14, d f=2, P<0.01\right)$ higher than among those tested negative (37.31\%), however, this association was highly confounded by age (Table 3 ). 


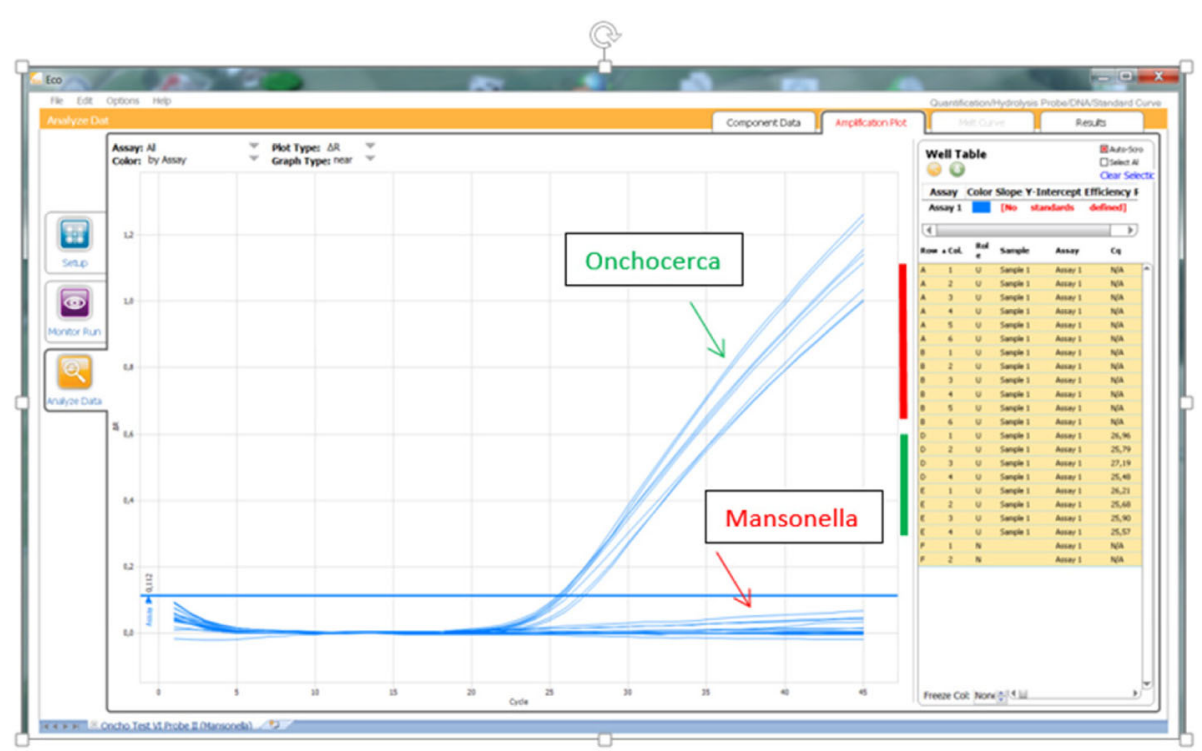

Fig. 1 Assessment of the specificity of novel O-5S qPCR assay in the detection of Onchocercia volvulus and Mansonella streptocerca DNA

\section{Risk factors and symptoms}

For the variables "distance of residence from river" (< $1 \mathrm{~km}: 84.96 \%$ / 74.63\%; > $1 \mathrm{~km}: 15.04 \%$ / 25.37\%) and "fetching water from river nearby residence" (Yes: 96.24\% / 94.03\%), no significantly different proportions were found among individuals tested positive and negative. For the variable "collecting wood from nearby forest" (Yes: $72.93 \%$ / 55.22\%), the proportion were significantly $\left(\chi^{2}=6.29, d f=1, P=0.01\right)$ higher among individuals tested positive than among those who tested negative. Also for the variables "palpable inguinal lymph nodes" (43.61\% / 20.90\%) and "skin itching around buttock" (74.44\% / 46.27\%), the proportions were significantly $\left(\chi^{2}=9.77, d f=1\right.$, and $\chi^{2}=15.46, d f=1$, respectively,
$P<0.01$ each) higher among individuals who tested positive than those who tested negative (Table 4).

Multivariate logistic regression has shown association only for age and itching of the skin with positive results of O-5S qPCR. Among those who tested positive, individuals were more likely 30 years or older than among individuals tested negative (adjusted OR $=2.17$; 95\% CI: 1.11-4.23). Among those who tested positive, individuals reported itching around buttocks more often than individuals tested negative (adjusted OR = 2.13; 95\% CI: 1.04-4.47) (Table 4).

\section{Discussion}

This is the first study assessing a novel O-5S quantitative real-time PCR for the molecular confirmation of $O$.

Table 3 Socio-demographic characteristics of clinically suspected onchocerciasis subjects along with O-5S qPCR assay

\begin{tabular}{|c|c|c|c|c|c|}
\hline Variable & & Study populationn (\%) & Positiven (\%) & Negativen (\%) & $P$-value ${ }^{a, b}$ \\
\hline Sample size & Patients & $200(100)$ & $133(100)$ & $67(100)$ & na \\
\hline \multirow[t]{2}{*}{ Gender } & Female & $44(22.00)$ & $30(22.56)$ & $14(20.90)$ & $0.79^{a}$ \\
\hline & Male & $156(78.00)$ & $103(77.44)$ & $53(79.10)$ & \\
\hline \multirow[t]{5}{*}{ Age (years) } & Range & $3-72$ & $3-72$ & $5-66$ & na \\
\hline & Median & 29 & 32 & 15 & na \\
\hline & Interquartile range & $12.75-45.00$ & $20-45$ & $10.0-35.5$ & na \\
\hline & Age group: 3-29 & $103(51.50)$ & $58(43.61)$ & $45(67.16)$ & $<0.01^{a *}$ \\
\hline & Age group: 30-72 & $97(48.50)$ & $75(56.39)$ & $22(32.84)$ & \\
\hline \multirow[t]{3}{*}{ Occupation } & Child, student & $72(36.00)$ & $33(24.81)$ & $39(58.21)$ & $<0.01^{\mathrm{a} *}$ \\
\hline & Farmer & $119(59.50)$ & $94(70.68)$ & $25(37.31)$ & $<0.01^{a *}$ \\
\hline & Other occupation & $9(4.50)$ & $6(4.51)$ & $3(4.48)$ & $1.00^{b}$ \\
\hline
\end{tabular}

${ }^{a}$ Chi-square tests

${ }^{\mathrm{b}}$ Fisher exact chi-square test

*Significant differences were defined as $P$-value $<0.05$

Abbreviation: na not applicable 
Table 4 Assessment of potential risk factors for onchocerciasis through O-5S qPCR assay

\begin{tabular}{|c|c|c|c|c|c|}
\hline$\underline{\text { Variable }}$ & & Study population $n(\%)$ & Positive $n(\%)$ & Negative $n(\%)$ & $P$-value ${ }^{a, b}$ \\
\hline Sample size & Patients & $200(100)$ & $133(100)$ & $67(100)$ & na \\
\hline \multirow[t]{2}{*}{ Distance of residence from river } & $<1 \mathrm{~km}$ & $163(81.50)$ & $113(84.96)$ & $50(74.63)$ & \multirow[t]{2}{*}{$0.08^{\mathrm{a}}$} \\
\hline & $>1 \mathrm{~km}$ & $37(18.50)$ & $20(15.04)$ & $17(25.37)$ & \\
\hline \multirow[t]{2}{*}{ Fetching water from river nearby residence } & Yes & $191(95.50)$ & $128(96.24)$ & $63(94.03)$ & \multirow[t]{2}{*}{$0.49^{b}$} \\
\hline & No & $9(4.50)$ & $5(3.76)$ & $4(5.97)$ & \\
\hline \multirow[t]{2}{*}{ Collecting wood from nearby forest } & Yes & $134(67.00)$ & $97(72.93)$ & $37(55.22)$ & \multirow[t]{2}{*}{$0.01^{a *}$} \\
\hline & No & $66(33.00)$ & $36(27.07)$ & $30(44.78)$ & \\
\hline \multirow[t]{3}{*}{ Palpable inguinal lymph nodes } & Not known & $2(1.00)$ & $1(0.75)$ & $1(1.49)$ & na \\
\hline & Yes & $72(36.00)$ & $58(43.61)$ & $14(20.90)$ & \multirow[t]{2}{*}{$<0.01^{\mathrm{a} *}$} \\
\hline & No & $126(63.00)$ & $74(55.64)$ & $52(77.61)$ & \\
\hline \multirow[t]{2}{*}{ Skin itching around buttock } & Yes & $130(65.00)$ & $99(74.44)$ & $31(46.27)$ & \multirow[t]{2}{*}{$<0.01^{a *}$} \\
\hline & No & $70(35.00)$ & $34(25.56)$ & $36(53.73)$ & \\
\hline
\end{tabular}

${ }^{\mathrm{a}}$ Chi-square tests

${ }^{\mathrm{b}}$ Fisher exact chi-square test

*Significant differences were defined as $P$-value $<0.05$

Abbreviation: na not applicable

volvulus infections in Goma district, Southwest Ethiopia, which is an endemic region for onchocerciasis. The present study showed that the newly developed assay is more sensitive than both microscopic examination of SSS and the conventional O-150 qPCR assay for the diagnosis of human onchocerciasis. This might be attributable to the implementation of a very stable hydrolysis probe that is $100 \%$ specific for $O$. volvulus from clinical samples. Moreover, the data presented in this study showed that the O-5S qPCR assay for the diagnosis of onchocerciasis from SSS can detect low-density DNA of $O$. volvulus. This is especially needed for reliable programmatic monitoring and evaluations regarding national elimination campaigns [31]. Furthermore, the novel assay was highly sensitive, approaching a lower limit of detection of six templates of the target sequence, which increased the detection limit by half in comparison to the O-150 qPCR [28]. This might be due to the careful choice of the target sequence with only moderate secondary structure forming primers, which prevent the formation of primer-primer dimers [29].

The results of the present study did not show any significant differences between microscopy and O-150 qPCR. These two tests had comparable positivity rates of $37 \%$ and $39 \%$, respectively. Furthermore, the concordance rate between microscopy and O-150 qPCR was high: 77\% among individuals tested positive by O-5S qPCR, 100\% among individuals tested negative by $\mathrm{O}-5 \mathrm{~S}$ qPCR, and $85 \%$ of all clinically suspected patients. These results indicate that the additional introduction of the O-150 qPCR would not substantially increase the diagnostic yield (13\%). Completely replacing microscopy by O-150 qPCR would have even decreased the diagnostic yield by $3 \%$ in this study. In contrast, application of the novel O-5S qPCR resulted in an additional diagnostic yield of 32\%. The O-5S qPCR had a much higher sensitivity in comparison to microscopy (56\%) or O-150 qPCR (59\%), whereas the two conventional tests had limited concordance rates of $71 \%$ and $73 \%$, respectively, compared to O-5S qPCR. Although the sensitivity of the two conventional tests is limited, the present study showed that both tests reached a specificity of $100 \%$ for O. volvulus.

Previously, different studies were conducted in various regions of Ethiopia to determine the prevalence of onchocerciasis using direct microscopy from SSS. In the present study, the positivity rate for microscopic detection of onchocerciasis was $37 \%$, a finding comparable to positivity rates as described in other studies from Ethiopia 31\% in Bebeka [36] and 34\% in Blue Nile valley [37]. On the other hand, the positivity rate of onchocerciasis in the present study was higher than reports from other locations in Ethiopia 17\% Gilgel Ghibe river valley [38], 23\% Kaso Hixi [39] and 22\% in Teppi area [40] and lower than a study conducted in Anfilo district (75\%) [1]. Of note, our finding has initiated the Zonal health bureau to launch ivermectin mass treatment which benefits not only the study participant but also other individuals living in the study area which was not included in the present study.

Among individuals tested positive by $\mathrm{O}-5 \mathrm{~S}$ qPCR, the prevalence of males (77\%) was higher than that of females (23\%). This finding is in line with previous studies that reported a higher proportion of males among patients with onchocerciasis $[1,33]$, which could be due to occupational exposure and susceptibility of study participants. In the rural parts of Ethiopia, males are more involved in outdoor activities than females and prefer to wear short pants while performing outdoor activities [1]. Thus, outdoor activities and wearing short pants may render males more prone to black fly's bites. 


\section{Conclusions}

The newly designed assay for the detection of O. volvulus DNA from SSS shows an improvement in performance and an important step towards appropriate diagnosis of onchocerciasis. This, in turn, can provide a crucial contribution to disease progress monitoring including reliable programmatic monitoring and evaluation of MDA, for example, to control the elimination of onchocerciasis. Consequently, future evaluation and demonstration trials are planned to apply the novel O-5S qPCR in endemic regions of Ethiopia. Moreover, the novel O-5S qPCR could serve as a valuable tool for future drug trials, especially if detection and quantification of low $\mathrm{mf}$ densities from tissue samples are required.

\section{Additional files}

Additional file 1: Text. Selection of $O$. volvulus $5 S$ sequence and alignment of target sequence from $O$. volvulus with related nematode sequences and Plasmodium falciparum. (DOCX $24 \mathrm{~kb}$ )

Additional file 2: Table S1. Additional file 2: Table S1. Socio-demographic, clinical and diagnostic data of the study subjects. (XLSX $224 \mathrm{~kb}$ )

Additional file 3: Figure S1. Calibration curve of the novel O5-S qPCR. (DOCX $22 \mathrm{~kb}$ )

Additional file 4: Figure S2. $X Y$-graph showing the correlation between O-150 qPCR and O-5S qPCR. (DOCX 19 kb)

\section{Abbreviations}

DITM: Department for Infectious Diseases and Tropical Medicine; ME: minimum evolution; mf: microfilariae; SSS: skin snip sample

\section{Acknowledgements}

The authors are very thankful for the study participants to take part in this study. The authors would like to acknowledge the health extension workers at the site of data collection for their nice mobilization of the community. Additionally, the authors thank Drs Kifle Dagne and Haileyesus Adamu from Addis Ababa University, Ethiopia, Dr. Bruktawit Kebede from Jimma University, Ethiopia, Mr. Wondwossen M. Yeshaw from the University of Groningen, the Netherlands for their constructive comments and fruitful discussions. Furthermore, the authors thank Agata Rhomberg for technical assistance at DITM.

\section{Funding}

This work was supported by the Graduate School of Addis Ababa University (to SAM), and by the Friedrich-Baur Foundation, the Ludwig-Maximilians University of Munich (to MB).

\section{Availability of data and materials}

All data generated or analyzed during this study are included in this published article as supplementary information files (Additional file 2: Table S1).

\section{Authors' contributions}

SAM, SA, KT, MB, TL, GB and KHH conceived the study. SAM, SA, AZ and MGA collected samples. SAM, SA, MG, MB, MS and FB performed the experiments. SAM, KT, MB, MS, SP and KHH analyzed the data. SAM, MB and $\mathrm{KHH}$ wrote the manuscript with contributions from all authors. All authors read and approved the final manuscript.

\section{Ethics approval and consent to participate}

The study protocol was reviewed by Jimma University, College of Public Health and Medical Sciences Ethical Review Committees (RPGC/09/2012) and approved by the Ministry of Science and Technology of Ethiopia (Ref. No 3-10/713/04). Before the enrollment of study participants, written informed consent was obtained from participants and parents in case of minors aged $<18$ years. Given illiteracy among rural populations, thumbprints were used as an alternative to signatures in accordance with the recommendations of the national Ethics
Committee. Participants were fully free to withdraw from the study without any consequences and without affecting the patients' rights to health care, medical advice, or treatment. Samples were shipped to the Department for Infectious Diseases and Tropical Medicine at the Ludwig-Maximilians-University in Munich, Germany based on an official "material transfer agreement."

\section{Consent for publication}

Not applicable.

\section{Competing interests}

The authors declare that they have no competing interests.

\section{Publisher's Note}

Springer Nature remains neutral with regard to jurisdictional claims in published maps and institutional affiliations.

\section{Author details}

'Department of Medical Laboratory Sciences and Pathology, College of Health Sciences, Jimma University, Jimma, Ethiopia. ${ }^{2}$ Department Microbial, Cellular and Molecular Biology, Addis Ababa University, Addis Ababa, Ethiopia. ${ }^{3}$ Department of Infectious Diseases and Tropical Medicine, Medical Center of the University of Munich, Munich, Germany. ${ }^{4}$ Dr. Battke SCIENTIA $\mathrm{GmbH}$, Taufkirchen, Germany. ${ }^{5}$ University Medical Center Hamburg-Eppendorf, University of Hamburg, Hamburg, Germany. ${ }^{6}$ German Centre for Infection Research (DZIF), partner site Munich, Munich, Germany.

Received: 5 January 2017 Accepted: 17 September 2017

Published online: 02 October 2017

\section{References}

1. Dori GU, Belay T, Belete H, Panicker KN, Hailu A. Parasitological and clinicoepidemiological features of onchocerciasis in west Wellega, Ethiopia. J Parasit Dis. 2012;36:10-8.

2. WHO. Working to overcome the global impact of neglected tropical diseases update 2011. Geneva; 2011.

3. Denery JR, Nunes AAK, Hixon MS, Dickerson TJ, Janda KD. Metabolomicsbased discovery of diagnostic biomarkers for onchocerciasis. PLoS Negl Trop Dis. 2010;4:e834.

4. WHO. Guidelines for stopping mass drug administration and verifying elimination of human onchocerciasis - criteria and procedures. Geneva: WHO; 2016.

5. Lustigman S, Prichard RK, Gazzinelli A, Grant WN, Boatin BA, McCarthy JS, et al. A research agenda for helminth diseases of humans: the problem of helminthiases. PLoS Negl Trop Dis. 2012;6:e1582.

6. Remme JHF. Research for control: the onchocerciasis experience. Trop Med Int Health. 2004;9(2):243-54.

7. Attah SK, Ayeh-Kumi PF, Sittie AA, Oppong IV, Nyarko AK. Extracts of Euphorbia hirta Linn. (Euphorbiaceae) and Rauvolfia vomitoria Afzel (Apocynaceae) demonstrate activities against Onchocerca volvulus microfilariae in vitro. BMC Complement Altern Med. 2013;13:66.

8. Federal Ministry of Health (FMoH). Guidelines for Onchocerciasis elimination in Ethiopia. Addis Ababa: Federal Ministry of Health (FMoH); 2014

9. Deribe K, Meribo K, Gebre T, Hailu A, Ali A, Aseffa A, et al. The burden of neglected tropical diseases in Ethiopia, and opportunities for integrated control and elimination. Parasit Vectors. 2012;5:240.

10. Murdoch ME. Onchodermatitis. Curr Opin Infect Dis. 2010;23:124-31.

11. Harnette W. DNA-based detection of Onchocerca volvulus. Trans R Soc Trop Med Hyg. 2002;96:S231-4.

12. Udall DN. Recent updates on onchocerciasis: diagnosis and treatment. Clin Infect Dis. 2007;44:53-60.

13. Rosenblatt JE. Laboratory diagnosis of infections due to blood and tissue parasites. Clin Infect Dis. 2009:49:1103-8.

14. Lloyd MM, Gilbert R, Taha NT, Weil GJ, Meite A, Kouakou IMM, et al. Conventional parasitology and DNA-based diagnostic methods for onchocerciasis elimination programmes. Acta Trop. 2015;146:114-8.

15. Boatin BA, Toé L, Alley ES, NJD N, Borsboom G, JDF H. Detection of Onchocerca volvulus infection in low prevalence areas: a comparison of three diagnostic methods. Parasitology. 2002;125:545-52.

16. Cook GC, Zumla Al, editors. Manson's tropical diseases. 22nd ed. Elsevier Health Sciences: Elsevier; 2009. 
17. Tang THT, López-Vélez R, Lanza M, Shelley AJ, Rubio JM, Luz SLB. Nested PCR to detect and distinguish the sympatric filarial species Onchocerca volvulus, Mansonella ozzardi and Mansonella perstans in the Amazon region. Mem Inst Oswaldo Cruz. 2010;105:823-8.

18. Hall LR, Pearlman E. Pathogenesis of onchocercal keratitis (river blindness). Clin Microbiol Rev. 1999:12:445-53.

19. Bradley JE, Nirmalan N, Kläger SL, Faulkner H, Kennedy MW. River blindness: a role for parasite retinoid-binding proteins in the generation of pathology? Trends Parasitol. 2001;17:471-5.

20. Hoerauf A, Brattig N. Resistance and susceptibility in human onchocerciasis beyond Th1 vs. Th2. Trends Parasitol. 2002;18:25-31.

21. Pischke S, Büttner DW, Liebau E, Fischer P. An internal control for the detection of Onchocerca volvulus DNA by PCR-ELISA and rapid detection of specific PCR products by DNA detection test strips ${ }^{\text {TM }}$. Trop Med Int Health. 2002;7:526-31.

22. Rokeach LA, Zimmerman PA, Unnasch TR. Epitopes of the Onchocerca volvulus RAL1 antigen, a member of the calreticulin family of proteins, recognized by sera from patients with onchocerciasis. Infect Immun. 1994; 62:3696-704.

23. Meredith SE, Unnasch TR, Karam M, Piessens WF, Wirth DF. Cloning and characterization of an Onchocerca volvulus specific DNA sequence. Mol Biochem Parasitol. 1989;36:1-10.

24. Meredith SEO, Lando G, Gbakima AA, Zimmerman PA, Unnasch TR. Onchocerca volvulus: application of the polymerase chain reaction to identification and strain differentiation of the parasite. Exp Parasitol. 1991;73: 335-44.

25. Zimmerman PA, Toe L, Unnasch TR. Design of Onchocerca DNA probes based upon analysis of a repeated sequence family. Mol Biochem Parasitol. 1993;58:259-67.

26. Vincent JA, Lustigman S, Zhang S, Weil GJ. A comparison of newer tests for the diagnosis of onchocerciasis. Ann Trop Med Parasitol. 2000;94:253-8.

27. König R, Nassri A, Meindl M, Matuja W, Kidunda AR, Siegmund V, et al. The role of Onchocerca volvulus in the development of epilepsy in a rural area of Tanzania. Parasitology. 2010;137:1559-68.

28. Fink DL, Fahle GA, Fischer S, Fedorko DF, Nutman TB. Toward molecular parasitologic diagnosis: enhanced diagnostic sensitivity for filarial infections in mobile populations. J Clin Microbiol. 2011:49:42-7.

29. Taylor S, Wakem M, Dijkman G, Alsarraj M, Nguyen M. A practical approach to RT-qPCR-publishing data that conform to the MIQE guidelines. Methods. 2010;50(4):S1-5.

30. Golden A, Faulx D, Kalnoky M, Stevens E, Yokobe L, Peck R, et al. Analysis of age-dependent trends in Ov16 lgG4 seroprevalence to onchocerciasis. Parasit Vectors. 2016;9:338

31. Thiele EA, Cama VA, Lakwo T, Mekasha S, Abanyie F, Sleshi M, et al. Detection of Onchocerca volvulus in skin snips by microscopy and real-time polymerase chain reaction: implications for monitoring and evaluation activities. Am J Trop Med Hyg. 2016;94:906-11.

32. Central Statistical Agency (Federal Democratic Republic of Ethiopia). The 2007 population and housing census of Ethiopia, result for Oromia region. Addis Ababa: Central Statistical Agency; 2010.

33. Benson DA, Karsch-Mizrachi I, Lipman DJ, Ostell J, Wheeler D. GenBank. Nucleic Acids Res. 2008:36:D25-30.

34. Beissner M, Awua-Boateng NY, Thompson W, Nienhuis WA, Klutse E, Agbenorku P, et al. A genotypic approach for detection, identification, and characterization of drug resistance in Mycobacterium ulcerans in clinical samples and isolates from Ghana. Am J Trop Med Hyg. 2010;83:1059-65.

35. Zarroug IMA, Hashim K, EIMubark WA, Shumo ZAI, Salih KAM, EINojomi NAA, et al. The first confirmed elimination of an onchocerciasis focus in Africa: Abu Hamed, Sudan. Am J Trop Med Hyg. 2016;95:1037-40.

36. Taticheff S, Abebe M, Workneh W, Hana NG. Onchocerciasis: a prevalence study in Bebeka, Ethiopa. Trop Med Parasitol. 1987;38:279-82

37. Jira C. Prevalence of onchocerciasis in Blue Nile valley of western Ethiopia. Indian J Public Health. 1993:37:135-7.

38. Taye A, Gebre-Michael T, Taticheff S. Onchocerciasis in Gilgel Ghibe river valley Southwest Ethiopia. East Afr Med J. 2000;77:116-20.

39. Dana D, Debalke S, Mekonnen Z, Kassahun W, Suleman S, Getahun K, et al. A community-based cross-sectional study of the epidemiology of onchocerciasis in unmapped villages for community directed treatment with ivermectin in Jimma zone, southwestern Ethiopia. BMC Public Health. 2015;1:1-7.

40. Legesse M, Balcha F, Erko B. Status of onchocerciasis in Teppi area, southwestern Ethiopia, after four years of annual community-directed treatment with ivermectin. Ethiop J Health Dev. 2010;24:51-6.

\section{Submit your next manuscript to BioMed Central and we will help you at every step:}

- We accept pre-submission inquiries

- Our selector tool helps you to find the most relevant journal

- We provide round the clock customer support

- Convenient online submission

- Thorough peer review

- Inclusion in PubMed and all major indexing services

- Maximum visibility for your research

Submit your manuscript at www.biomedcentral.com/submit
Biomed Central 